\title{
Adesão ao uso de anticoagulante oral cumarínico por pacientes portadores de fibrilação atrial
}

RESUMO | Objetivo: avaliar a adesão do paciente com Fibrilação Atrial em Terapia com anticoagulante oral cumarínico. Método: Estudo descritivo-exploratório, transversal e prospectivo com intervenção não randomizado de abordagem quantitativa, realizado com 40 participantes, aplicou-se o Escore de Adesão Simonetti e a Escala de Avaliação Analógica Visual e após a intervenção educativa para análise de Time in Therapeutic Range sob CAAE: 79973017.1.0000.5462. Resultado: Identificouse que 5\% apresentaram risco baixo de adesão, $60 \%$ risco médio e $35 \%$ risco alto. Após análise dos oito meses do TTR pré e pós-intervenção, não houve mudança significativa entre os períodos ( $p=0,638)$. Conclusão: Evidenciou-se que o grupo de baixa adesão manteve melhor tempo na faixa terapêutica relacionado ao médio e alto risco. Indicativo este de maior impacto da intervenção educativa realizada pelo enfermeiro.

Palavras-chaves: Fibrilação Atrial; Anticoagulantes; Adesão à Medicação.

ABSTRACT|Objective: to evaluate the adherence of the patient with Atrial Fibrillation in Therapy with oral coumarin anticoagulant. Method: Descriptive-exploratory, cross-sectional and prospective study with a non-randomized intervention with a quantitative approach, carried out with 40 participants, the Simonetti Adherence Score and the Visual Analog Evaluation Scale were applied and after the educational intervention for analysis of Time in Therapeutic Range under CAAE: 79973017.1.0000.5462. Result: It was identified that $5 \%$ had low risk of adherence, $60 \%$ medium risk and $35 \%$ high risk. After analyzing the eight months of TTR before and after intervention, there was no significant change between the periods ( $p=0.638)$. Conclusion: It was evidenced that the low adherence group maintained a better time in the therapeutic range related to medium and high risk. Indicative of greater impact of the educational intervention carried out by the nurse.

Keywords: Atrial Fibrillation; Anticoagulants; Medication Adherence.

RESUMEN | Objetivo: evaluar la adherencia del paciente con fibrilación auricular en terapia con anticoagulante cumarínico oral. Método: Estudio descriptivo-exploratorio, transversal y prospectivo con una intervención no aleatorizada con abordaje cuantitativo, realizado con 40 participantes, se les aplicó el Simonetti Adherence Score y la Escala de Evaluación Visual Analógica y luego de la intervención educativa para el análisis del Tiempo en el Rango Terapéutico bajo CAAE: 79973017.1.0000.5462. Resultado: Se identificó que $5 \%$ riesgo bajo de adherencia, $60 \%$ riesgo medio y $35 \%$ riesgo alto. Después de analizar los ocho meses de TTR antes y después de la intervención, no hubo cambios entre los períodos $(p=0,638)$. Conclusión: Se evidenció que el grupo de baja adherencia mantuvo el mejor tiempo en el rango terapéutico relacionado con riesgo medio y alto. Indicativo de mayor impacto de la intervención educativa realizada por la enfermera.

Palabras claves: Fibrilación Atrial; Anticoagulantes; Cumplimiento de la Medicación.

\section{Tatiane Roberto de Sousa Borges}

Enfermeira, Residente em Enfermagem Cardiovascular no Instituto Dante Pazzanese de Cardiologia, São Paulo - Brasil. ORCID: 0000-0002-9924-1677

\section{Márcia de Fátima da Silva Meyer}

Enfermeira, Mestre em Enfermagem EEUSP, Enfermeira no Instituto Dante Pazzanese de Cardiologia, São Paulo - Brasil. ORCID: 0000-0001-8944-5492

\section{Sérgio Henrique Simonetti}

Enfermeiro, Coordenador do Programa de Residência em Enfermagem Cardiovascular. Coordenador do Núcleo de Enfermagem do Programa Residência Multiprofissional. Serviço de Educação Continuada. Pós-Doutorado em Ciências pelo IDPC-USP. Doutor e Mestre em Ciências pela EEUSP. MBA Executivo em Administração: Gestão de Saúde pela FGV. Educação e Tecnologia pela UFSCAR. Gestão Pública e Informática em Saúde pela UNIFESP. Residência Enfermagem Cardiovascular pelo Instituto Dante Pazzanese de Cardiologia, São Paulo - Brasil.

ORCID: 0000-0001-7840-8004
INTRODUÇÃO

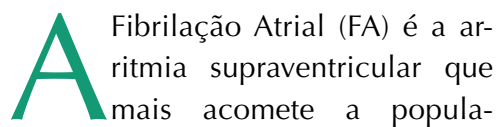
ção. Caracteriza-se por uma desordem no impulso elétrico atrial, o que causa despolarizações atriais de forma descoordenada, com consequente perda da capacidade de contração dessas câmaras cardíacas. ${ }^{(1,2)} \mathrm{O}$ acúmulo de sangue nos átrios causado por essa deficiência na sístole atrial, expõe o individuo a formação de trombos. ${ }^{(2)}$

As taxas de incidência e prevalência de FA vêm crescendo com o passar dos anos, o que tem relação com o envelhecimento da população, sexo, aumento das morbidades e piora do estilo de vida..$^{(1,3)}$ 
A associação entre a FA e o risco para fatores tromboembólicos é bem conhecida, o processo tromboembólico ocorre a partir da tríade de Virchow que engloba três fatores: estase sanguínea atrial; lesão endotelial e o aumento da trombogenicidade sanguínea, própria da FA. As causas para esse processo são multifatoriais, e uma vez os trombos formados nas câmaras atriais, eles se deslocam para a circulação sistêmica, sendo o cérebro a região mais afetada, ocasionando o Acidente Vascular Encefálico (AVE). ${ }^{(1)}$

A anticoagulação oral é um método de escolha para o tratamento desse público, com o objetivo de prevenir fenômenos tromboembólicos, incluindo os AVEs isquêmicos (AVEi). A classe de anticoagulante mais utilizada é a dos cumarínicos que compreendem os antagonistas da vitamina $\mathrm{K}$ podendo ser representados pela warfarina e femprocumona. ${ }^{(4)}$

O monitoramento do tratamento com Anticoagulantes Orais (ACO) é realizado a partir do exame de Tempo de Protrombina (TP) que significa o tempo de formação do coágulo de fibrina, após indução em ambiente laboratorial. O TP é expresso através do RNI (Razão Normalizada Internacional), método utilizado em todo o mundo com o objetivo de padronizar o resultado da análise do TP. ${ }^{(5-6)} \mathrm{O} R \mathrm{RI}$ ideal estabelecido para os pacientes com FA é entre 2,0 e 3,0.(1)

Para avaliar a qualidade do tratamento com ACO, utiliza-se o cálculo do Time in Therapeutic Range (TTR) ou tempo na faixa terapêutica no período analisado para um determinado grupo de pacientes. ${ }^{(7)}$

Para calcular o TTR um dos testes utilizados é o método de interpolação linear de Rosendaal ${ }^{(8)}$, o qual permite calcular o número de dias em intervalo terapêutico, a partir de uma relação linear entre dois valores consecutivos de RNI com atribuição de um valor específico de RNI para cada dia. Portanto, o cálculo da porcentagem de dias em intervalo terapêutico é dado pela razão entre a diferença de dois valores consecutivos de RNI dentro do intervalo terapêutico, dividida pela diferença total entre eles ${ }^{(7)}$.

O valor de TTR ideal para os usuários de $\mathrm{ACO}$ é de no mínimo 70\%, com esse valor os benefícios da anticoagulação são mais evidentes, como a prevenção de AVE. ${ }^{(9)}$

Manter-se na faixa terapêutica é um desafio para o paciente, já que o uso de ACO cumarínico e a estabilidade dos níveis de RNI estão relacionados a diversos fatores como à adesão ao tratamento, idade, comorbidades, interações com outros medicamentos, aspectos genéticos e ingesta de alimentos ricos em vitamina $K^{(5,10)}$ Outros aspectos como, o nível de cognição, emocionais e psicológicos também afetam a estabilidade do RNI na faixa terapêutica. ${ }^{(11)}$

A Organização Mundial da Saúde (OMS) define adesão à terapia medicamentosa relacionada à medida que o comportamento de uma pessoa (tomar medicação, seguindo uma dieta, e/ou executando mudanças de estilo de vida), corresponde às recomendações acordadas de um profissional de saúde". ${ }^{(12)}$

A educação em saúde para esclarecimento da importância da terapêutica, do modo de uso da medicação, identificação e prevenção de efeitos adversos, interações medicamentosas e interferência da dieta, é a principal ferramenta para obter níveis satisfatórios de adesão à terapeutica e consequente redução da mortalidade desses pacientes que fazem uso prolongado de ACO. ${ }^{(11)}$

Objetivou com este estudo avaliar a adesão do paciente com FA em Terapia com Anticoagulante Oral (T-ACO) cumarínico por meio da pergunta de pesquisa: "qual a adesão dos pacientes com FA em uso de ACO cumarínico?".

\section{MÉTODO}

Trata-se de um estudo descritivo-exploratório, transversal e prospectivo com intervenção não randomizado de abor- dagem quantitativa. Realizado com usuários de ACO atendidos em ambulatório especializado em Eletrofisiologia de um Hospital Público de grande porte especializado em Cardiologia do Estado de São Paulo.

A população deste estudo foi composta pelos pacientes portadores de FA em uso de ACO com seguimento na instituição no período de janeiro 2018 a janeiro de 2019. A seleção da amostra foi de forma aleatória, recrutando os pacientes no momento de espera dos resultados de RNI. Os pacientes foram convidados e orientados sobre os objetivos do estudo e iniciada após aceitação e assinatura do Termo de Consentimento Livre e Esclarecido de acordo com os critérios de elegibilidade: idade acima de 18 anos; alfabetizados (sabe ler e escrever); pacientes portadores de FA em T-ACO (Warfarina e Femprocumona) há no mínimo um ano.

Para a coleta foi aplicado um instrumento para caracterização dos aspectos sociodemográficos dos participantes e o Escore de Adesão Simonetti ${ }^{(13)}$ construído para avaliar adesão de paciente em tratamento com ACO, além da Escala Analógica Visual (EAV) ${ }^{(14)}$ que permite a auto avaliação do paciente e também a percepção do enfermeiro referente à adesão terapêutica.

O Escore de Adesão Simonetti foi construído para o público em T-ACO para avaliar a adesão desses usuários ao tratamento conforme figura 1. O escore permite a avaliação da adesão do usuário de ACO pelas seguintes variáveis: Uso inadequado, Procedimento invasivo, Interação Medicamentosa, Hábitos Alimentares, Condições Clínicas e Outros Fatores (aumento ou ganho de peso; estresse; tabagismo dentre outros). ${ }^{(13)}$

Sua pontuação foi calculada a partir do odds ratio de regressão múltipla. Em seguida, os fatores intervenientes foram categorizados com base no escore obtido como baixo ( $\leq 10$ pontos), médio (11 a 30) e alto ( $\geq 31$ pontos) para o evento $\mathrm{RNI}$ normal, conforme figura 1. Com C 
Statistic de 0,940 (IC (95\%) = 0,920 0,960; $\mathrm{p}<0,001)$ demonstrando bom desempenho para discriminar a ocorrência do evento ou não. ${ }^{(13)}$

A Escala Analógica Visual tem como finalidade avaliar a adesão auto percebida do usuário com relação a T-ACO, atribuindo uma pontuação de 0-10 para seu nível de adesão ao tratamento conforme figura 2, o que caracteriza o auto relato, método muito utilizado para avaliar e coletar informações sobre adesão tanto na prática em saúde quanto na pesquisa. (14) Em contrapartida o enfermeiro pesquisador atribuiu sua percepção referen- te ao participante da pesquisa, também utilizando a pontuação da escala.

Assim, aplicou-se o instrumento sociodemográficos, e logo após o Escore de Adesão Simonetti e, consequentemente a EAV. Após a identificação dos fatores intervenientes a adesão a T-ACO o enfermeiro pesquisador realizou a intervenção educativa, com a finalidade de sanar dúvidas e fazer orientações conforme a necessidade de cada usuário e fatores identificados pelo Escore Simonetti (Uso inadequado, Procedimento invasivo, Interação Medicamentosa, Hábitos Alimentares, Condições Clínicas e

Figura 1 - Pontuação Escore de Adesão Simonetti ${ }^{(13)}$

\begin{tabular}{|c|c|c|c|}
\hline \multicolumn{4}{|c|}{$\begin{array}{l}\text { ESCORE DE ADESAOO SIMONETTI PARA USUÁRIOS DE } \\
\text { ANTICOAGULANTE ORAL }\end{array}$} \\
\hline FATORES INTERVENIENTES & SIM & NÃo & ESCORE \\
\hline - Interaçăo Medicamentosa & 0 & 30 & $\cdots$ \\
\hline - Uso Inadequado & 0 & 18 & $\cdots$ \\
\hline - Hábitos Alimentares & 0 & 07 & $\cdots$ \\
\hline - Procedimentos Invasivos & 0 & 02 & $\cdots$ \\
\hline - Condiçðes Clínicas/Problemas de Saúde & 0 & 02 & $\cdots$ \\
\hline - Outros Fatores & 0 & 05 & $\cdots$ \\
\hline TOTAL & \multicolumn{2}{|c|}{$(0 a 64)$} & 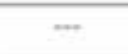 \\
\hline \multicolumn{4}{|l|}{ Daixo ( $\leq 10$ pontos) } \\
\hline \multicolumn{4}{|l|}{ Medio (11 a 30 pontos) } \\
\hline$D$ Alto $(\geq 31$ pontos) & & & \\
\hline
\end{tabular}

Figura 2 - Escala Analógica Visual (14)

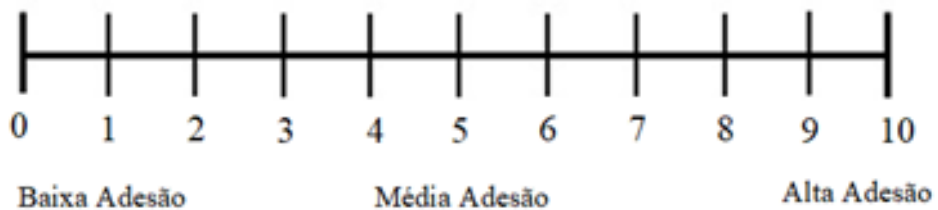


na cidade de São Paulo. Prevaleceram 75\% com renda salarial de 1 a 3 salários mínimos.

Em relação aos antecedentes de saúde $85 \%$ referem apresentar Hipertensão Arterial Sistêmica (HAS), 42,5\% Diabetes Mellitus (DM), 35\% Dislipidemia (DLP). Dentre outros problemas de saúde, destacaram-se 7,5\% para Infarto Agudo do Miocárdio, o Hipotireoidismo e a Trombose. O anticoagulante cumarínico em uso por $100 \%$ dos participantes foi a Warfarina, com tempo médio de uso de 43 meses.

Constatou-se que não há relação significativa entre ter alta, média ou baixa adesão com as variáveis sociodemograficas, conforme tabela 1, já que apresentaram um $\mathrm{p} \geq 0,05$ quando se verificou respectivo evento.

Pelo Escore de Adesão Simonetti identificou-se no item de interação medicamentosa que $90 \%$ dos participantes têm histórico de interação diante do uso de alguma medicação contínua ou intermitente. As medicações que interagem com o anticoagulante cumarínico mais encontradas no uso entre os participantes da pesquisa foram amiodarona $\mathbb{R} 20(50 \%)$, sinvastatina ${ }^{\circledR} 19(47,5 \%)$, omeprazol ${ }^{\circledR} 7$ $(17,5 \%)$, anti-inflamatório cinco $(12,5 \%)$ e levotiroxina ${ }^{\circledR} \operatorname{cinco}(12,5 \%)$.

Em uso inadequado $70 \%$ negaram, e $30 \%$ relataram, dentre estes que relataram, o fator que se destacou foi o esquecimento, ou seja, não tomou a dose diária, com 17,5\% dos participantes.

Relacionado a hábitos alimentares apenas $12,5 \%$ mostraram variações ou mudanças radicais na alimentação, sendo que 7,5\% após início do tratamento com anticoagulante cumarínico retiraram totalmente de sua dieta os alimentos ricos em vitamina $K$, pois em algum momento receberam essa orientação.

O que se refere a procedimentos invasivos, apenas $12,5 \%$ informou ser submetido algum evento nos últimos meses. Em relação a condições clínicas/problemas de saúde $22,5 \%$ relataram resfriado, diarreia e febre, em 2,5\% dos participantes. Dentro de outros fatores $47,5 \%$ dos participantes relataram, estresse $27,5 \%, 17,5 \%$ alte- ração de peso corporal, 7,5\% consumo de álcool e 5\% afirmaram ser fumante.

Após análise das variáveis do Escore de Adesão Simonetti atribuindo as de-

Tabela 1. Relação de variáveis e Adesão (Escore Adesão Simonetti). São Paulo, Brasil 2020.

\begin{tabular}{|c|c|c|c|c|c|c|}
\hline \multirow[t]{2}{*}{ Variáveis } & \multirow[t]{2}{*}{ Categorias } & \multicolumn{2}{|c|}{$\begin{array}{c}\text { Adesão } \\
\text { Baixa ou } \\
\text { Média }\end{array}$} & \multicolumn{2}{|c|}{$\begin{array}{c}\text { Adesão } \\
\text { Alta }\end{array}$} & \multirow[t]{2}{*}{ p-valor } \\
\hline & & $\mathbf{N}^{\circ}$ & $\%$ & $\mathbf{N}^{\circ}$ & $\%$ & \\
\hline \multirow{2}{*}{ Sexo } & Feminino & 12 & 46,2 & 5 & 35,7 & \multirow{2}{*}{0,739} \\
\hline & Masculino & 14 & 53,8 & 9 & 64,3 & \\
\hline \multirow{4}{*}{ Estado Civil } & Solteiro & 4 & 15,4 & 0 & 0 & \multirow{4}{*}{0,252} \\
\hline & Casado & 17 & 65,4 & 12 & 85,7 & \\
\hline & Divorciado & 3 & 11,5 & 0 & 0 & \\
\hline & Viúvo & 2 & 7,7 & 2 & 14,3 & \\
\hline \multirow{7}{*}{ Escolaridade } & Sabe ler escrever & 1 & 3,8 & 0 & 0 & \multirow{7}{*}{0,786} \\
\hline & Ensino Fundamental Incompleto & 13 & 50 & 6 & 42,9 & \\
\hline & Ensino Fundamental Completo & 4 & 15,4 & 1 & 7,1 & \\
\hline & Ensino Médio Incompleto & 0 & 0 & 1 & 7,1 & \\
\hline & Ensino Médio Completo & 6 & 23,1 & 5 & 35,7 & \\
\hline & Ensino Superior Incompleto & 1 & 3,8 & 0 & 0 & \\
\hline & Ensino Superior Completo & 1 & 3,8 & 1 & 7,1 & \\
\hline \multirow{2}{*}{ Trabalho } & Não & 18 & 69,2 & 9 & 64,3 & \multirow{2}{*}{0,356} \\
\hline & $\operatorname{Sim}$ & 8 & 30,8 & 5 & 35,7 & \\
\hline \multirow{2}{*}{ Aposentado(a) } & Não & 9 & 34,6 & 5 & 35,7 & \multirow{2}{*}{1,000} \\
\hline & $\operatorname{Sim}$ & 17 & 65,4 & 9 & 64,3 & \\
\hline \multirow{2}{*}{ Moradia } & Própria & 24 & 92,3 & 14 & 100 & \multirow{2}{*}{0,533} \\
\hline & Alugada & 2 & 7,7 & 0 & 0 & \\
\hline \multirow{2}{*}{$\begin{array}{l}\text { Local em que } \\
\text { reside }\end{array}$} & São Paulo & 20 & 76,9 & 11 & 78,6 & \multirow{2}{*}{1,000} \\
\hline & Outras cidades de São Paulo & 6 & 23,1 & 3 & 21,4 & \\
\hline \multirow{5}{*}{ Renda } & Menos de 1 salário mínimo & 0 & 0 & 1 & 7,1 & \multirow{5}{*}{0,314} \\
\hline & 1 até 3 salários mínimos & 21 & 80,8 & 9 & 64,3 & \\
\hline & Mais de 3 até 5 salários mínimos & 4 & 15,4 & 3 & 21,4 & \\
\hline & Mais de 5 até 7 salários mínimos & 1 & 3,8 & 0 & 0 & \\
\hline & Mais de 9 salários mínimos & 0 & 0 & 1 & 7,1 & \\
\hline \multirow{2}{*}{ HAS } & Não & 4 & 15,4 & 2 & 14,3 & \multirow{2}{*}{1,000} \\
\hline & $\operatorname{Sim}$ & 22 & 84,6 & 12 & 85,7 & \\
\hline \multirow{2}{*}{ DM } & Não & 15 & 57,7 & 8 & 57,1 & \multirow{2}{*}{1,000} \\
\hline & $\operatorname{Sim}$ & 11 & 42,3 & 6 & 42,9 & \\
\hline \multirow{2}{*}{ DLP } & Não & 14 & 53,8 & 12 & 85,7 & \multirow{2}{*}{0,081} \\
\hline & Sim & 12 & 46,2 & 2 & 14,3 & \\
\hline
\end{tabular}

Fonte: Elaboração própria do autor, 2020. vidas pontuações, observou-se que 5\% representa risco baixo de adesão, 60\% risco médio de adesão e 35\% apresentaparticipantes tiveram escore $\leq 10$ o que 
ram escore $\geq 31$ que é condizente a risco alto de adesão.

Com a EAV identificou-se que 27,5\% dos participantes atribuíram-se de 8 a 10 pontos relacionados à adesão ao tratamento. Para a percepção do enfermeiro pesquisador quanto à aplicação da EAV houve uma maior variabilidade de conceitos, as notas mais frequentes foram de oito para $22,5 \%$ dos participantes e nota sete para $20 \%$ dos participantes, a nota mais baixa atribuída foi quatro para 5\% deles.

Após análise do TTR pré e pós oito meses da intervenção educativa, evidenciou-se que não houve mudança significativa do TTR pós em relação ao TTR pré $(p=0,638)$. Entre os grupos (baixa/média/ alta adesão) observa-se que não houve diferença significativa do TTR $(p=0,437)$, mostrando uma estabilidade entre eles.

Porém, observou-se que o grupo de baixa adesão mostrou um ganho maior no TTR em relação aos outros, indicativo de que a intervenção educativa teve um maior impacto neste grupo com média de $14,28 \%$ e desvio padrão de 20,91\%. Entretanto não foi estatisticamente significante, o que pode estar relacionado ao número baixo de participantes dentro deste grupo. Já os outros dois grupos (média e alta adesão) apresentaram médias próximas $(2,28 \%$ e $2,92 \%)$ e desvio padrão (36,66\% e $24,55 \%)$, respectivamente.

Realizou-se a correlação do TTR com a nota do participante $(r=-0,049$ e $p$ valor de $0,763)$, a nota do pesquisador $(r=-0,105$ e $p=0,517)$ e com o Escore de Adesão Simonetti ( $r=-0,085 p=0,602)$, que não constatou correlação significativa entre aqueles que tiveram notas altas e escore de adesão alto, com o aumento do TTR.

Com esses resultados, optou-se por outra análise retirando os participantes com TTR pré $>80 \%$, pois observou que o grupo com TTR abaixo deste valor teria mais ganho pós-intervenção educativa. Restaram 34 pacientes com TTR até $80 \%$, que constatou uma maior proximidade da significância com $(p=0,098)$ quando comparado com a análise feita com o total de participantes $(p=0,638)$, o que corrobora que aqueles com menor TTR pré tendem a obter maiores resultados de TTR positivos quando há intervenção educativa.

\section{DISCUSSÃO}

Nota-se que em 2010 houve um aumento significativo das taxas de prevalência da FA com o aumento da idade, apresentando taxas entre os $\geq 35$ anos de ambos os sexos maiores que o dobro da prevalência geral que é de 596,2 para homens e 373,1 entre mulheres (por cada 100.000 habitantes). ${ }^{(3)}$ Uma tendência que reforça o encontrado neste estudo, já que a faixa de idade variou entre 42 e 83 anos com predomínio do sexo masculino. Isso nos remete as mudanças epidemiológicas que acomete a população com FA, com o envelhecimento da população e a

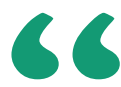

\section{Já as comorbidades} como Hipertensão Arterial Sistêmica, Diabetes, Dislipidemia, entre outras relatadas pelos participantes deste estudo, também não mostraram associação significativa com adesão ao tratamento. predisposição do sexo masculino levando assim a necessidade do T-ACO. ${ }^{(1)}$

Em relação à escolaridade nota-se que houve um maior número de participantes com ensino fundamental incompleto $47,5 \%$, o que se assemelha com outros trabalhos que apontam um maior número de participantes com 5 anos de estudos, ${ }^{(15-16)}$ o que destaca um perfil de baixa escolaridade desta população. Já em relação ao perfil socioeconômico alguns estudos sobre a temática demonstram faixa salariais de até 1 salário mínimo, como em um estudo que do total de 39 participantes, $71,79 \%$ apresentaram de 0 a 1 salário mínimo. ${ }^{(15)}$ Já em outro, dos 100 pacientes entrevistados $73 \%$ apresentavam renda de até 1 salário mínimo. ${ }^{(17)}$ Fato este que difere do encontrado em nosso estudo em que apenas 2,5\% participante apresentou renda abaixo de 1 salário mínimo.

Pode-se constatar que as variáveis descritivas como (sexo, estado civil, escolaridade, o fato de trabalhar e/ou ser aposentado, moradia, renda) não tiveram relação significativa com ter alta, média ou baixa adesão, o que pode ser explicado pelo fato da adesão ao tratamento ter uma maior interferência, não só por esses fatores e da adesão à medicação, como também de hábitos de vida diária, interação medicamentosa, fatores externos e emocionais do paciente. ${ }^{(11,18)}$

Já as comorbidades como Hipertensão Arterial Sistêmica, Diabetes, Dislipidemia, entre outras relatadas pelos participantes deste estudo, também não mostraram associação significativa com adesão ao tratamento. Já o que foi demonstrado em outro estudo é a ausência de associação significativa com níveis e estabilidade de INR. ${ }^{(10)}$

Os fatores analisados pelo Escore de Adesão Simonetti, utilizado neste estudo têm exatamente o intuito de analisar a adesão ao tratamento como um todo, considerando todas as variáveis que interferem na adesão, não somente a questão da adesão à medicação, mas também aquelas relacionadas aos hábitos de vida diário que estão ligados ao aspecto comportamental de cada paciente. ${ }^{(13)}$ 
O fator de interação medicamentosa neste estudo demonstrou uso por $12,5 \%$ dos participantes de anti-inflamatório em alguns casos sem prescrição médica ou comunicado ao setor ambulatorial de anticoagulação. Estudos que analisa os fatores intervenientes na adesão, quando questionados sobre o uso de medicamentos sem prescrição médica, grande parte $77,8 \%$ dos participantes declararam que apenas utilizavam medicamentos quando indicado pelo médico. ${ }^{(18-19)}$

Quando se ressalta o que leva ao uso inadequado do anticoagulante oral, o esquecimento ganhou destaque, dentre os $30 \%$ que pontuaram neste quesito, $17,5 \%$ deles relataram esquecimento. Já em um estudo que abordou a adesão ao anticoagulante mediante uso do instrumento para avaliar adesão - Medida de Adesão ao Tratamento (MAT), apenas um participante relatou esquecimento dentre o total de 27 integrantes da pesquisa. ${ }^{(18)}$ Em outro estudo sobre a variação de (RNI), que também avaliou a adesão ao anticoagulante a partir do teste de Morisky, 64,0\% relataram uso regular do medicamento, respeitando dose e horários. ${ }^{(20)}$

Relacionado à interação alimentar houve evidencias que demonstraram interferência na efetividade da T-ACO, em um estudo $81,8 \%$ de 118 participantes demonstraram ter conhecimento de como deveria ser a alimentação, considerando a interação entre a Warfarina e os alimentos ricos em vitamina K. ${ }^{(21)}$

Há um método utilizado com os usuários de ACO de uma instituição especializada em cardiologia de um estudo anterior, que consiste em orientações sobre interações da warfarina com alimentos e medicamentos, avaliação periódica do RNI e notificação do serviço pelo paciente quando há alteração da dieta ou quando ocorre algum evento adverso como o sangramento. ${ }^{(20)}$ Já a instituição deste estudo oferta essas informações ao paciente no início do tratamento com o anticoagulante e nos retornos para avaliação periódica do RNI, nestes momentos há o esclarecimento de dúvidas e de pontuações quando ne- cessário ou quando observa-se o valor de RNI fora da faixa terapêutica.

Em relação às notas atribuídas pelos participantes sobre adesão autopercebida e a percepção do pesquisador, percebe-se predominância de conceitos altos entre os pacientes, e uma maior variabilidade de conceitos entre as notas do pesquisador. Em um estudo feito com uma população também portadora de uma condição crônica que é a HAS, e que também avaliou a percepção de adesão na visão do paciente e do profissional, dos 12 pacientes, 6 se deram a pontuação máxima cinco que os apontam como aderidos ao tratamento, não contendo aderidos. Já na visão do profissional, apenas três pacientes foram pontuados com pontuação cinco considerados em adesão e quatro pacientes como não aderidos. ${ }^{(22)}$ Isso mostra que a percepção do paciente sobre si tende ser superestimada, já a do

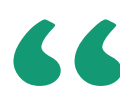

\section{Relacionado à interação alimentar houve evidencias que demonstraram interferência na efetividade da T-ACO, em um estudo $81,8 \%$ de 118 participantes demonstraram ter conhecimento de como deveria ser a alimentação}

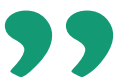

profissional que tem um olhar mais crítiCo, analítico e ponderado.

Sabe-se que o valor de um TTR significativo para prevenir doenças tromboembólicas como o AVE é (TTR>70\%) e, corrobora em um estudo de coorte realizado em 4 países europeus, constatou que o TTR médio variou de $66 \%$ em Itália a $72,6 \%$ no Reino Unido, já na França e Alemanha menos de $50 \%$ dos pacientes se classificaram com um (TTR>70\%). Os eventos de AVE e sangramentos foram evidenciados como frequentes nos pacientes que tiveram um $\mathrm{TTR} \leq 70 \%$. ${ }^{(23)}$

O fato da análise do TTR pré e pós-intervenção educativa não ter apresentado mudança significativa dentre os 40 participantes, pode estar relacionado ao nível de absorção dos pacientes em relação às orientações passadas no início do tratamento e o que realmente é executado por eles durante a terapêutica. ${ }^{(11)}$

Neste estudo a análise indicou, mesmo sem significância estatística, um ganho maior do TTR no grupo de baixa adesão, indicando um benefício maior deste grupo com a atividade educativa. Estudo que avaliou entre outros itens a adesão ao tratamento daqueles que fazem uso de anticoagulante oral, concluiu-se que aqueles que já fizeram ou estavam em uso do anticoagulante a um período de tempo, ou seja, já eram familiarizados com o tratamento, a estratégia educativa não se mostrou eficiente. ${ }^{(24)}$ Com isso pode constatar que quando há um contato prévio com a rotina de tratamento, há um ganho menos significativo com a intervenção educativa. ${ }^{(25)}$

A limitação neste estudo destacou-se o tempo de coleta de dados restrito que impactou significativamente, na amostra, não repercutindo nos resultados diante do objetivo proposto, sugerindo assim, um estudo longitudinal com uma amostra expansiva.

\section{CONCLUSÃO}

O estudo possibilitou conhecer o perfil de adesão dos pacientes envolvi- 
dos, identificar os fatores interferentes na adesão mais comuns na amostra estudada, além da percepção referente à adesão de ambas as partes, autopercebida e do enfermeiro.

Mesmo que não tenha ocorrido significância estatística, temos uma discreta evidência que o grupo identificado de baixa adesão teve maior benefício com a in- tervenção educativa desenvolvida quando reconhecido os fatores intervenientes da adesão através do escore aplicado.

Diante disso, há necessidade do enfermeiro conhecer o perfil dos usuários de anticoagulantes orais e, empoderar-se nas tomadas de decisões em implementar e inovar intervenções educativas.

Assim, as estratégias educativas reali- zadas pelo enfermeiro na prática clínica são essenciais na promoção de adesão e na prevenção de eventos adversos relacionados ao uso de anticoagulante oral cumarínico, com impactos favoráveis na melhoria da adesão ao tratamento e em mudanças de hábitos com foco na manutenção do TTR da Relação Normalizada Internacional recomendada.

\section{Referências}

1. Magalhães LP, Figueiredo MJO, Cintra FD, Saad EB, Kuniyoshi RR, Teixeira RA, et al. Il Diretrizes Brasileiras de Fibrilação Atrial. Arq. Bras. Cardiol. 2016;106(4), Supl 2:1-22. Disponível em: http://dx.doi.org/10.5935/abc.20160055

2. Quilici A, Bento AM, Ferreira FG, Cardoso LF, Moreira RSL, Silva SC. Enfermagem em Cardiologia. $2^{\text {a }}$ Ed. São Paulo: Atheneu;2014.p:492-93.

3. Kirchhof $P$, Benussi $S$, Kotecha D, Ahlsson A, Atar D, Casadei B, et al. ESC Scientific Document Group, 2016 ESC Guidelines for the management of atrial fibrillation developed in collaboration with EACTS European Heart Journal. 2016. 37(38);2893-962. Disponível em: https://doi.org/10.1093/eurheartj/ ehw210

4. Molina FT, Júnior GZ. Anticoagulantes cumarínicos: ações, riscos e monitoramento da terapêutica. SaBios: Rev. Saúde e Biol. 2014;9(2):75-82. Disponível em:http://revista.grupointegrado.br/revista/index.php/sabios2/article/ view/1263/599

5. Clé DV, Garcia AA, Brunetta DM, Schwartzmann PV, Moriguti JC. Anticoagulação em pacientes hospitalizados. Medicina (Ribeirão Preto. Online) 2010;43(2):107-17. Disponível em: https://doi.org/10.11606/issn.2176-7262. v43i2p107-117

6. Araújo ACO, Domingues RB, Bellen van B. Determinação do INR: comparação entre método convencional e dispositivo portátil. J. Vasc. Bras. 2014;13(2):8893. Disponível em: http://dx.doi.org/10.1590/jvb.2014.051.

7. Guedes M, Rego C. Estudo HIPOGAIA: monitorização da hipocoagulação oral com dicumarínicos no concelho de Gaia. Rev Port Cardiol. 2016;35(9):45965. Disponível em: https://doi.org/10.1016/..repc.2016.03.003

8. Rosendaal FR, Cannegieter SC, van der Meer FJ, Briet E. A method to determine the optimal intensity of oral anticoagulant therapy. Thromb Haemost. 1993;69(3):236-9. https://openaccess.leidenuniv.nl/bitstream/handle/1887/1793/303_324.pdf

9. Camm AJ, Lip GY, De Caterina R, Savelieva I, Atar D, Hohnloser SH, et al; ESC Committee for Practice Guidelines (CPG). 2012 focused update of the ESC Guidelines for the management of atrial fibrillation: An update of the 2010 ESC Guidelines for the management of atrial fibrillation. European Heart Journal. 2012; 33(21):2719-47. https://doi.org/10.1093/eurheartj/ehs253

10. Ávila CW, Aliti GB, Feijó MKF, Rabelo ER. Pharmacological adherence to oral anticoagulant and factors that influence the international normalized ratio stability. Rev Latino-AmEnfermagem.2011;19(1):18-25 Disponivel em: http://www.scielo.br/scielo.php?script=sci_arttext\&pi$d=S 0104-11692011000100004 \&$ lng=en.

11. Esmerio FG, Souza EN, Leiria TL, Lunelli R, Moraes MA. Uso crônico de anticoagulante oral: implicações para o controle de níveis adequados. Arq Bras Cardiol.2009;93(5):549-54. Disponível em: http://www.scielo.br/scielo.php?script=sci_arttext\&pid=S0066-782X2009001100017\&lng=en.

12. World Health Organization. Adherence to long-term therapies. Evidence for action.2003. Disponível em: http://apps.who.int/iris/bitstream/10665/42682/1/9241545992.pdf

13. Simonetti SH, Faro ACM, Bianchi ERF Escore de adesão para usuários de anticoagulantes orais. Int J Cardiovasc Sci.2018;31(4):383-92. Disponível em: http://publicacoes.cardiol.br/portal/ijcs/portugues/2018/v3104/pdf/3104009.pdf 14. Polejack L, Seidl EMF. Monitoramento e avaliação da adesão ao tratamento antirretroviral para HIV/aids: desafios e possibilidades. Ciênc. saúde coletiva.
2010; 15(Supl1):1201-08. Disponível em: http://dx.doi.org/10.1590/S141381232010000700029 .

15. Neto OPA, Cunha CM, Rodrigues CM, Resende TC. Perfil clínico, adesão e satisfação terapêutica de pacientes em uso de anticoagulantes orais. Rev. Aten. Saúde, São Caetano do Sul.2016;14(47):61-6. Disponível em: http://dx. doi.org/10.13037/ras.vol14n47.3389

16. Souza TF, Colet CF, Heineck I. Nível de informação e adesão à terapia de anticoagulação oral com varfarina em pacientes acompanhados em ambulatório de atenção primária à saúde. J Vasc Bras.2018;17(2):109-16. Disponível em: https://doi.org/10.1590/1677-5449.012017

17. Figueirêdo TR, Nascimento MO, Silveira MMBM, Costa CRB, Queiroga AV, Bezerra SMMS. Conhecimento de pacientes em acompanhamento ambulatorial sobre a terapia de anticoagulação oral. J. res.: fundam. care. Online.2016; 8(1):3883-92. Disponível em: http://www.seer.unirio.br/index.php/cuidadofundamental/article/viewFile/5162/pdf_1806

18. Enzweiler LL, Rodrigues DM, Carvalho ARS. Anticoagulação oral: conhecendo os fatores de adesão e não-adesão a terapia. Anais do $5^{\circ}$ Seminário Nacional Estado e Políticas Sociais, Universidade Estadual do Oeste do Paraná, 2011. Disponível em: http://cac-php.unioeste.br/projetos/gpps/midia/seminario6/arqs/Trab_completos_politicas_seguridade/Anticoagulacao_oral_fatores_adesao.pdf

19. Simonetti SH, Faro ACM, Bianchi ERF. Clinical predictors involved in adhesion of oral anticoagulant users. Nursing in focus.2019;10(1),02-06. Disponivel em: https://pesquisa.bvsalud.org/portal/resource/pt/biblio-1028045

20. Kitahara ST, Silva EA, Fagundes DJ, Costa LMA, Ferraz RF, Costa FAA. Avaliação da Variação de Razão Normalizada Internacional em Pacientes Anticoagulados através de Metodologia Diferenciada. Rev Bras Cardiol.2014;27(5):342-8. Disponível em: http://www.onlineijcs.org/english/sumario/27/pdf/v27n5a08.pdf

21. Nery RT, Pimenta MC, Costa JM, Marins MAP, Machado CJ. Identificação de fatores interferentes no controle da anticoagulação em um ambulatório multiprofissional. Revista Intercâmbio.2016:7:191-207. Disponível em: http://www. intercambio.unimontes.br/index.php/intercambio/article/view/94/82

22. Coutinho FHP, Sousa IMC. Percepção dos indivíduos com hipertensão arterial sobre sua doença e adesão ao tratamento medicamentoso na estratégia de saúde da família. Rev. Baiana de Saúde Pública. 2011;35(2):397-411. Disponível em: http://rbsp.sesab.ba.gov.br/index.php/rbsp/article/view/314/281 23. Cotté FE, Benhaddi H, Duprat-Lomon I, Doble A, Marchant N, Letierce A, Huguet M. Vitamin K Antagonist Treatment in Patients With Atrial Fibrillation and Time in Therapeutic Range in Four European Countries. Clin Ther. 2014;1;36(9):1160-8. Disponível em: http://www.clinicaltherapeutics.com/ article/S0149-2918(14)00456-1/pdf

24. Corbi ISA. Efeitos de um programa educativo na qualidade de vida relacionada à saúde e na adesão à terapia de anticoagulação oral: estudo clínico randomizado [teses]. Ribeirão Preto: Escola de Enfermagem de Ribeirão Preto; 2014. Disponivel em: http://www.teses.usp.br/teses/disponiveis/22/22132/tde22052014-163837/en.php, doi:10.11606/T.22.2014.tde-22052014-163837.

25. Simonetti SH, Massa VC, França JíD. Conventional and innovative educational method for oral anticoagulation user learning. Nursing in focus.2018;8(4):03-06. Disponivel em: 10.21675/2357-707X.2017.v8.n4.938 